\title{
PRESSURE INDUCED ELECTRONIC AND NaCl TO CsCI T YPE TRANSITIONS IN SOME RARE EARTH MONOTELLURIDES
}

\author{
A.K. Singh, A. Jayaraman ${ }^{*}$ and A. Chatterjee \\ Materials Science Division, National Aeronautical Laboratory, Bangalore-17, India
}

(Received 14June 1971 hy N.B. Hannay)

\begin{abstract}
High pressure X-ray diffraction study reveals that PrTe, SmTe and EuTe which possess $\mathrm{NaCl}$ type structure transform to CsCl type at $90 \pm 10,110 \pm 10,100 \pm 10 \mathrm{kbar}$ respectively. Prior to this transformation SmTe shows an anomalous decrease in volume with pressure, which is attributed to the $4 \mathrm{f}-5 \mathrm{~d}$ electronic transition.
\end{abstract}

RARE earth monochalcogenides crystallize in NaCl-type structure ${ }^{1}$ and are either metallic or semiconducting depending on the valence state of the rare earth ion; metallic if trivalent and semiconducting if divalent. ${ }^{2.3} \mathrm{Sm}, \mathrm{Eu}$ and $\mathrm{Yb}$ have a stable divalent state and hence their compounds are semiconducting. Also $\mathrm{Tm}$ in TmTe is divalent and the compound is semiconducting. They have anomalously large lattice constants, in the series of rare earth chalcogenides; these exhibit a systematic decrease in the lattice constant with increasing atomic number of the rare earth ion. Recent high pressure studies on EuTe ${ }^{4}, \mathrm{SmTe}$, $^{5}$ $\mathrm{TmTe}^{6}, \mathrm{SmSe}^{7}$ and $\mathrm{SmS}^{7}$ have revealed that under Pressure the rare earth ion undergoes an electronic transition from the divalent to the trivalent state. Resistivity measurements under pressure have ndicated that such a transition takes place continuously in TmTe, SmTe and SmSe and discontinuously in $\mathrm{SmS}$ at about $6.5 \mathrm{kbar}$. In these transformations the $\mathrm{NaCl}$-type structure is retained but the lattice parameter contracts by about 4 per cent.

In the present study we have investigated by nigh pressure X-ray diffraction. PrTe, SmTe and EuTe and find a pressure induced phase trans* ingation from $\mathrm{NaCl}$ to CsCl-type structure near

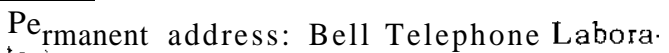
tories, Murray Hill, New Jersey, USA. about 100 kbar. In SmTe, prior to this transformation, the lattice parameter undergoes an anomalous decrease with pressure, due to continuous $4 f-5 d$ electron promotion. We believe that a pressure induced transition from $\mathrm{NaCl}$ type structure should be a common feature among the rare earth monochalcogenides.

Samples were prepared by reacting in a quartz tube stoichiometric amounts of high purity $\mathrm{Te}$ and the rare earth metal at $700^{\circ} \mathrm{C}$ and subsequent melting of the product in a sealed tantalum container kept in vacuum. The material thus obtained was polycrystalline with large single crystal regions. The $\mathrm{NaCl}$-type structure and the lattice constant were verified from X-ray powder diffraction data. SmTe and EuTe had conductivities in the range $1000 \Omega \mathrm{cm}$, while PrTe with Pr in the trivalent state, exhibited metallic conductivity.

High pressure X-ray studies were carried out using a diamond anvil camera of the type described by Bassett, Takahashi and Stook. ${ }^{8}$ Molybdenum radiation was used to record the powder patterns. The pressure generated was estimated using $\mathbf{A g}$ as internal standard. Powder pattern of the sample was recorded at zero applied pressure and then at several increasing pressures up to $150 \mathrm{kbar}$. In the lower pressure ranges data were collected using McWhan-Bond ${ }^{9}$ camera with $\mathrm{NaCl}$ 


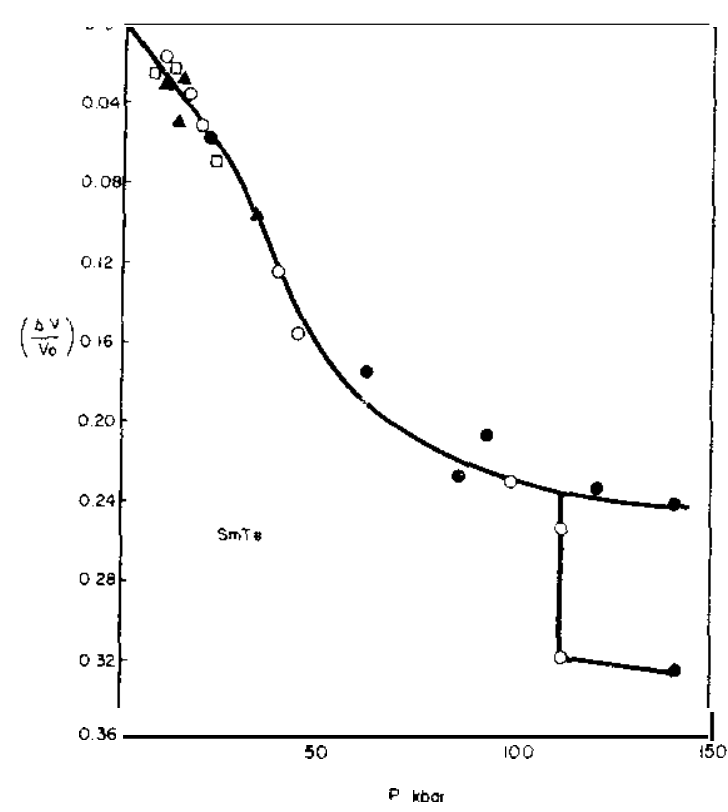

FIG. 1. The compression curve for SmTe obtained from high pressure X-ray diffraction data. and o - data points from diamond anvil camera with a silver marker; $\mathrm{c}$ - with $\mathrm{NaCl}$ marker. $\rightarrow$ McWhan-Bond camera with $\mathrm{NaCl}$ marker.

marker. A pattern recorded after the release of the pressure indicated that the changes observed were reversible.

In Fig. 1 the $\Delta V / V_{0}$ obtained from $X$-ray data is plotted against pressure for SmTe. In the pressure range $1-100 \mathrm{kbar}$, the volume shows an anomalous decrease with pressure. No change in the structure is observed in this pressure range, it continues to retain the $\mathrm{NaCl}$-type structure. The lattice parameter however, undergoes an anomalo's decrease with pressure and the volume decrease at $100 \mathrm{kbar}$ is about 24 per cent. We attribute the observed abnormal compression to a change in the valence state of the $\mathrm{Sm}^{2+}$ to $\mathrm{Sm}^{3+}$ with pressure, due to $4 f-5 d$ electron promotion. Our pressure volume data indicate that this electronic transition is spread over $\mathbf{3}$ broad pressure range. In this respect the pressure-volume behaviour 15 consistent with earlier resistance measurements ${ }^{5,7}$ which indicated a continuous $4 f-5 d$ electron promotion.

A new set of lines are observed in the X-ray photographs at pressures above $110 \mathrm{kbar}$. These are the diffraction lines from $a$ high pressure ph which is identified as having the Cscl-type stru ture. The diffraction lines, from both NaCl and CsCl phases co-exist over a small pressure rang At about $150 \mathrm{kbar}$, only the lines from the C.SCl phase arc observed. In Fig. I the discontinuitv volume at about $110 \mathrm{kbar} 1 \mathrm{~s}$ doe to the phase trap sition from $\mathrm{NaCl}$ to the CsCl-type structure. The volume change associated with this transition 1 s about 9 per cent.

Europiun telluride undergoes $\mathrm{NaCl}$ to $\mathrm{CsCl}$ type phase transition at about $100 \mathrm{kbar}$. The for of the compression curve seems to be differont from that observed in SmTe and does not suggest any appreciable conversion of $\mathrm{Eu}$ " to $\mathrm{Eu}^{3}{ }^{3}$ befor $\mathrm{NaCl}-\mathrm{CsCl}$ type transition takes place. The $\mathrm{AV} / \mathrm{V}_{\mathrm{O}}$ at $80 \mathrm{kbar}$ is about 14 per cent. Our initial compressibility is in fair agreement with the com. pressibility measured by Levy and Wachter.

In the case of PrTe, wherein $\mathrm{Pr}$ is in the valent state, the NaCi-type phase has a much smaller compressibility compared to the $\mathrm{NaCl}$ phase of SmTe and EuTe. For instance, $\triangle V / V_{0}$ at 90 kbar is 4.5 per cent. The $\mathrm{NaCl}$-type phase of PrTe exhibits a volume discontinuity at 90 픈 $10 k b a r$ due to $a$ phase transition to the CsCl-tym structure. The volume change associated with this transition is about 11.5 per cent.

Rooymans ${ }^{4}$ "has investigated EuTe as weiii as $\mathrm{SmTe}$ at high pressures and has reported an isostructural transition in both. The transition was attributed to a change in the valence state of Eu and Sm to the trivalent state. Further, Rooymans 4 11has suggested that the above trant-

sition occurs discontinuously near 30 and $40 \mathrm{kban}$ in EuTe and $\mathrm{SmTe}$ respectively. In the case of $\mathrm{SmTe}$, both with regard to the mode of occurrever of this transition and the pressure ranges, our data are in variance with Rooymans. Our X-ray data suggest that the electronic transition in SmTe takes place over a broad pressure range. With regard to EuTe, our high pressure $X-r$ iz dat suggest that no appreciable conversion of $\mathrm{Eu}^{2-}$ to $\mathrm{Eu}^{3+}$ takes place in the $\mathrm{NaCl}$ phase.

The main results of our work are summariset in Table 1. 
eEE⿱

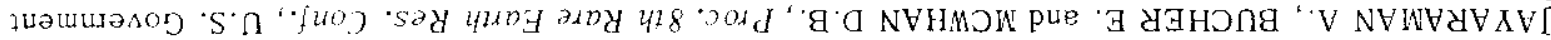
$\cdot(0 \angle 6 \mathrm{I})$

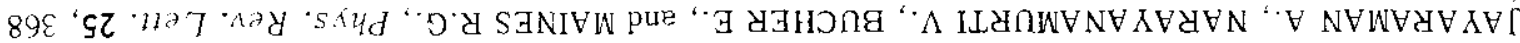

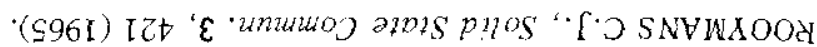

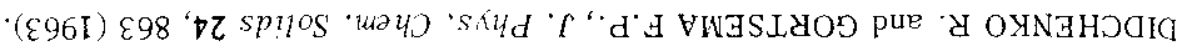

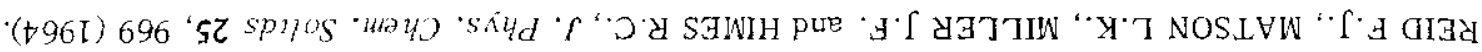

-dísmoliaj e jo pieme aył joj notjepuno

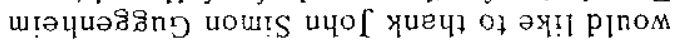

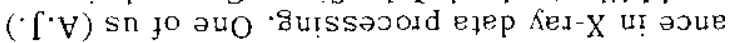
-7sisse dof mad eysn $\mathrm{S} \cdot \mathrm{s}$ in pue suorssnostp

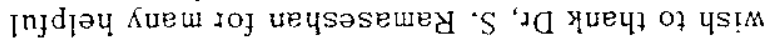

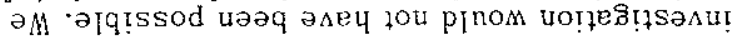

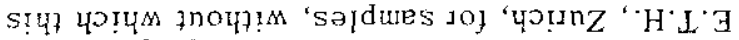

'YIS

' yosng $b$ ford pue Kassa [ MaN 'II!H KesinN

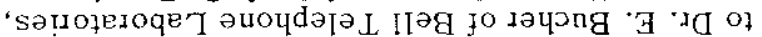

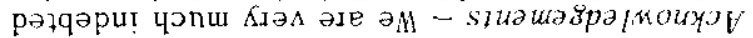

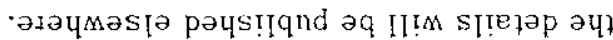

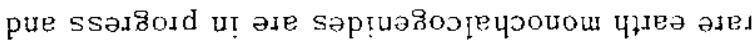

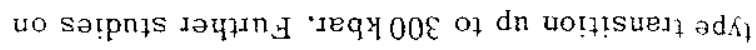
-

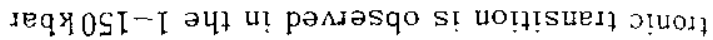

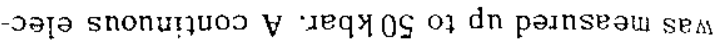

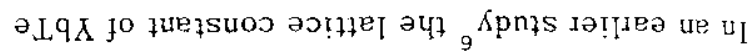

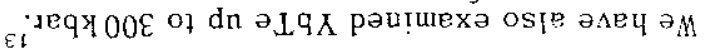

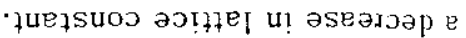

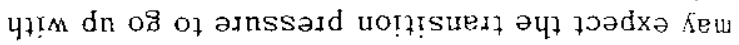

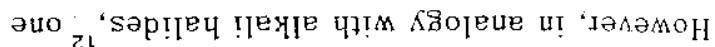

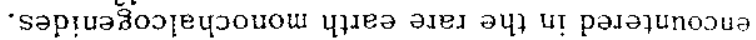

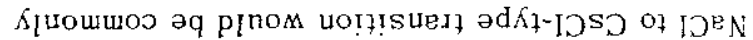

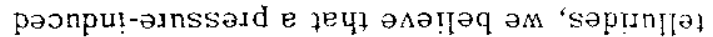

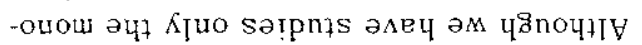

saseyd loso pire

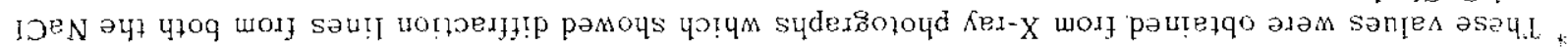

\begin{tabular}{|c|c|c|c|}
\hline $0 \mathrm{I} \div 00 \mathrm{I}$ & $0 I \mp 0 I I$ & $01 \mp 06$ & IDSD- Den fo annssajd วLlL \\
\hline$\% 9 \%$ II - & 6 & \%SII & 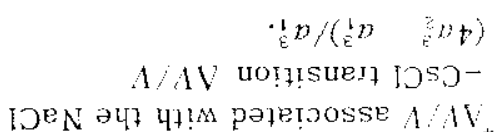 \\
\hline$\forall 10^{\circ} 0 \mp S \subseteq L^{\circ} \varepsilon$ & $\forall 10^{\circ} 0+959^{\circ} \varepsilon$ & $\forall 10 \cdot 0 \mp\left[9 L^{\circ} \mathcal{E}\right.$ & 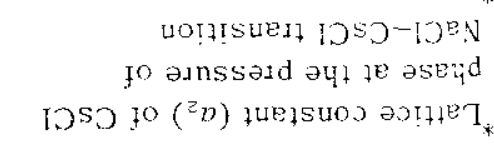 \\
\hline$\forall 20 \cdot 0=2 I Z \cdot 9$ & $\forall 20^{\circ} 0+266^{\circ} \mathrm{S}$ & $\forall Z 0^{\circ} 0: 8 I Z^{\circ} 9$ & 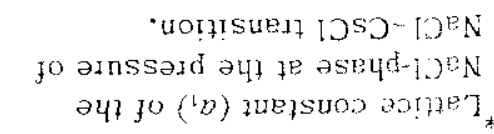 \\
\hline $\mathrm{yz00^{ \circ }}+\mp 165^{\circ} 9$ & $\forall Z 00^{\circ} 0 \mp S 6 S^{\circ} 9$ & $\forall 700^{\circ} 0 \div$ SIE. 9 & $\begin{array}{l}\text { anssad oidaydsohipe je } \\
\text { aseyd-ion jo quejsuoo arjeri }\end{array}$ \\
\hline
\end{tabular}

$$
\partial_{L^{n}}
$$$$
\partial_{\text {LW }}
$$$$
\partial_{L}{ }^{I} \mathrm{~d}
$$

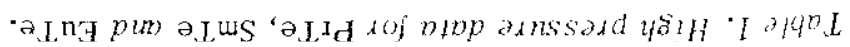


7. JAYARAMAN A., NARAYANAMURTI V., BUCHER E. and MAINES R.G., Phys. Rev. Lett. 25, 1430 (1970).

8. BASSETT W.A., TAKAHASHI T. and STOCK P.W., Rev. scient, Instrm. 38, 37 (1967).

9. MCWHAN D.B. and BOND W.L., Rev. scient. Instrum. 35, 626 (1964).

10. LEVY F. and WACHTER P., Solid Slate Commun. 8, 183 (1970).

11. ROOYMANS C.J.M., Ber. Bunsenges. Phys. Chem. 70, 1036 (1966).

12. See KLEMENT W., Jr., and JAYARAMAN A., Rev. Article in Solid State Chem. Vol. 3, Academic Press, (1966).

13. Results obtained by us -10 be published.

Hochdruck-Rontgenbeugung untersuchungen ergeben. daß PrTe, SmTe and EuTe, die eine NaCl-artige Struktur besitzen, verwandeln sich bei $90 \pm 10,110 \pm 10 \mathrm{bzw} .100 \pm 10 \mathrm{kbar}$ in eine CsCl-artige Struktur. Vor dieser Verwandlung zeigt SmTe mit zunehmendem Dmck eine anomale Volumenverminderung auf, die auf den $4 f-5 d$ Elektronendbergang zurdckgefiihrt wird. 